\title{
Anti-shielding Effect and Negative Temperature in Instantaneously Reversed Electric Fields and Left-Handed Media
}

\author{
Jian-Qi Shen * \\ Center for Optical and Electromagnetic Research, State Key Laboratory of Modern Optical Instrumentation, College of \\ Information Science and Engineering; Zhejiang Institute of Modern Physics and Department of Physics, Zhejiang University, \\ Hangzhou 310027, P. R. China
}

(August 30, 2018)

\begin{abstract}
The connections between the anti-shielding effect, negative absolute temperature and superluminal light propagation in both the instantaneously reversed electric field and the left-handed media are considered in the present paper. The instantaneous inversion of the exterior electric field may cause the electric dipoles into the state of negative absolute temperature and therefore give rise to a negative effective mass term of electromagnetic field ( i. e., the electromagnetic field propagating inside the negative-temperature medium will acquire an imaginary rest mass ), which is said to result in the potential superluminality effect of light propagation in this anti-shielding dielectric. In left-handed media, such phenomena may also arise.
\end{abstract}

PACS Ref: 78.20.Nv; 05.20.-y; 78.20.Ci

\section{INTRODUCTION}

This paper is concerned with the physically interesting anti-shielding effects of electric and magnetic dipoles, and the related phenomena such as the negative absolute temperature of dipole systems and the consequent superluminal light propagation. In nature, neither of the above three effects ( phenomena) is easily observed. Although negative magnetic permeability has been shown to be possible when a polariton resonance exists in the permeability, such as in the antiferromagnets $\mathrm{MnF}_{2}$ and $\mathrm{FeF}_{2}$ [1], or certain insulating ferromagnets [2], a negative permeability $(\mu)$ with low losses coexisting with a negative electric permittivity $(\epsilon)$ has not been demonstrated [3]. This means that in these magnetic anti-shielding materials $(\mu<0, \epsilon>0)$, electromagnetic wave cannot propagate through it, since the negative magnetic permeability and positive electric permittivity may result in an imaginary index of refraction. The principal reason for the state of negative absolute temperature being rarely observed in nature lies in that the attainment of negative temperature should be restricted to some critical conditions [4] such as that the system must possess an upper limit to the possible energy level of the allowed states; and the system should be thermally isolated from other system at positive temperature, for if a negative- and positive- temperature systems are in thermal contact, energy will be given up from the negative temperature to the positive, and therefore the negative-temperature system will decay to a normal state ( positive temperature ) through infinite temperature. In history, only the experiments of nuclear spin systems of some crystals, where the state of negative absolute temperature might be attained, were carried out $[5,6]$.

What is referenced above is in connection only with the anti-shielding effect and negative temperature of magnetic media; the light propagation in electric and magnetic anti-shielding media is not involved in these investigations. Here, however, we take into account the anti-shielding effect of electric media and the light propagation inside it, i. e., we deal with the statistical mechanics of the state of negative temperature caused by the instantaneous reversal of the exterior electric field and, based on this, briefly discuss the negative effective rest mass term of light due to negative temperature and the consequent superluminality effect in the anti-shielding dielectric. Here the negative effective rest mass term of light means that the electromagnetic field propagating inside the anti-shielding medium would acquire an imaginary rest mass. Although it sounds somewhat surprising that in the anti-shielding dielectric there exists the superluminal light propagation, this superluminality motion associated with the particle velocity rather than with the group velocity of the light field is actually familiar to us, since it is in connection with the "tachyon " representation in the Poincaré group [7] and is therefore said to be theoretically natural.

*E-mail address: jqshen@coer.zju.edu.cn 
In the present paper, the potential fact that the negative absolute temperature of dipole systems of left-handed media is also demonstrated. Left-handed media is such a hypothetical material which has a simultaneously negative electric permittivity and magnetic permeability. In 1968, Veselago [8] predicted that electromagnetic plane wave in a left-handed medium would propagate in a direction opposite to that of the flow of energy. It is of interest that the refractive index, $n$, of this medium is also negative, which can be rigorously derived by analyzing the boundary conditions of incident waves on the interface between left-handed and ordinary media. This, therefore, means that on the interface between air and left-handed media, anomalous refraction takes place [8,9]. It is apparently seen that in this media where both permittivity and permeability are taken to be negative, the anti-shielding effect arises.

We hold that there exists close relationship between the electromagnetic anti-shielding effect and the negative absolute temperature as well as the potential superluminal light propagation due to anti-shielding effects. The superluminal light propagation presented in this paper results from the statistical fluctuations of polarization charge in negative-temperature media, as will be discussed in Sec. 3. Since in the present paper we concern ourselves much with the light propagation in media, in next section we propose a phenomenological theory of effective rest mass term of light field, which is helpful in considering the light propagation in anti-shielding and negative-temperature media. The viewpoint of effective rest mass of electromagnetic field in media is useful in giving the vivid descriptions of some physical effects, for example, the Meissner effect in superconductivity can be interpreted as follows: the superconducting electrons, which produce the self-induced charge current [10], can provide the electromagnetic field with an effective rest mass $\left(m_{e f f}\right)$, and this nonvanishing $m_{e f f}$ is therefore responsible for the fact that the magnetic field penetrates only a short distance ( proportional to $m_{e f f}^{-1}$ ) within the surface of a superconductor. The interaction of light wave with media is one of the leading subjects in classical electrodynamics and quantum optics, where a multitude of physical processes can often be phenomenologically described by useful models at classical and/or quantum level [11-13].

\section{EFFECTIVE REST MASS TERM OF LIGHT FIELD}

In history, possibility of a light pulse with speed greater than that $(c)$ in a vacuum has been extensively investigated by many authors [14-19]. The various experiments with microwave signals performed by Nimtz et al. revealed superluminal velocities of evanescent modes [20-22]. Wang et al. used the method of gain-assisted linear anomalous dispersion to demonstrate the superluminal light propagation in the atomic caesium gas and measured a negative group-velocity index of the medium that means the faster-than- $c$ propagation of light [23,24]. We suggest that, based on the theory of effective rest mass of light field, Wang's superluminal propagation due to the anomalous dispersion in the gaseous atomic medium [23] can be phenomenologically understood as follows:

The relation between the frequency $\omega$ and wave vector $k$ of lightwave propagating inside the dispersive medium may be $\frac{\omega^{2}}{k^{2}}=\frac{c^{2}}{n^{2}}$ with $n$ being the optical refractive index. For highly absorptive media, the corresponding modification of this method can also apply. So, for convenience, here we are not ready to deal with the case of absorptive materials. This dispersion relation $\frac{\omega^{2}}{k^{2}}=\frac{c^{2}}{n^{2}}$ yields

$$
\frac{\omega^{2}}{k^{2}}=\frac{c^{2}}{1-\frac{c^{4}}{\hbar^{2}} \frac{m^{2}}{\omega^{2}}}
$$

with the effective rest mass, $m$, of the light being so defined that $m, n$ and $\omega$ together satisfy the following relation

$$
\frac{m^{2} c^{4}}{\hbar^{2}}=\left(1-n^{2}\right) \omega^{2}
$$

This, therefore, implies that the particle velocity of light ( photons ) is $v=n c$ and the phase velocity of light is $v_{p}=\frac{c}{n}=\frac{c^{2}}{v}$. Since this relation is familiar to us, we thus show that the above calculation is self-consistent, at least for the de Broglie wave ( particle ).

As an illustrative example of the application of the above formulation, we now evaluate the effective rest mass of lightwave propagating in some certain media. For instance, we consider the case of electron plasma in which the relative permittivity $\epsilon(\omega)$ reads $[25]$

$$
\epsilon(\omega)=1-\frac{\omega_{p}^{2}}{\omega^{2}}, \quad \omega_{p}^{2}=\frac{N\left(e^{*}\right)^{2}}{m \epsilon_{0}}
$$

with $\omega_{p}, N, e^{*}, m$ and $\epsilon_{0}$ being the electron plasma frequency, number of electrons per unit volume, effective charge of electrons, electron mass and electric permittivity in a vacuum, respectively. Note that here the electron plasma is 
regarded as cold plasma where the thermal effects can be ignored. Since the relative magnetic permeability, $\mu$, of this plasma can be approximately taken to be 1 and in consequence the refractive index $n(\omega)=\sqrt{\epsilon(\omega)}$, it follows from (2.2) and (2.3) that the effective rest mass of the incident electromagnetic wave $\left(\omega>\omega_{p}\right)$ is of the form

$$
m_{e f f}=\frac{\hbar \omega_{p}}{c^{2}}
$$

which is independent of the frequency of the electromagnetic wave propagating inside this plasma. It is of interest that the effective $\epsilon(\omega)$ of the array of long metallic wires (ALMWs) [26-29] ( that is now used to manufacture the

left-handed materials ) behaves as that of plasma, which means that in ALMWs media $\left(\epsilon(\omega)=1-\frac{\omega_{0}^{2}}{\omega^{2}}, \mu=1\right)$, the lightwave may also acquire an $\omega$ - independent effective rest mass. However, in most dielectric, e. g., the anomalous dispersive media [14] where the permittivity $\epsilon(\omega)$ cannot be taken to be the plasma type, the propagating lightwave may therefore acquire an $\omega$ - dependent effective rest mass. In what follows we calculate the group velocity in order to consider the superluminal light propagation in anomalous dispersive media.

It follows that the group-velocity index is

$$
n_{g}=\frac{1}{n}-\frac{m c^{3}}{\hbar^{2} k} \frac{\mathrm{d} m}{\mathrm{~d} \omega}
$$

which can yield the familiar formula

$$
n_{g}=n+\omega \frac{\mathrm{d} n}{\mathrm{~d} \omega},
$$

so long as we substitute the expression for the effective rest mass $m$ into the expression (2.5). Thus, the group velocity of light pulse is

$$
v_{g}=\frac{c}{n_{g}}=\frac{n c}{1-\frac{m n c^{3}}{\hbar^{2} k} \frac{\mathrm{d} m}{\mathrm{~d} \omega}} .
$$

This, therefore, means that if the rest mass $m$ is independent of $\omega$, then the particle velocity $v=n c$ equals the group velocity $v_{g}$, which is analogous to the case of the de Broglie particle; nevertheless, if the effective rest mass $m$ of the light in the dispersive medium depends strongly on $\omega$ ( e. g., the property caused in the anomalous-dispersive medium ), then the group velocity $v_{g}$ is completely divorced from the particle velocity $v$ and may be greater than $c$ or even negative. Here both the particle velocity and the group velocity refer to those of the same matter wave that possesses the wave-particle duality. Note, however, that although the group velocity $v_{g}$ of light propagation exceeds $c$ of the speed of light in a vacuum, this superluminal phenomenon is not related closely to the special relativity since in this case the particle velocity of photons does not often necessarily exceed $c$. In the present paper, from the point of view of effective rest mass we suggest a new possible superluminality effect of light in the negative-temperature medium ( i. e., the dielectric placed in the instantaneously reversed electric fields ). Since it is connection with the particle velocity, rather than with the group velocity measured in Wang's superluminal experiment, this superluminal light propagation in the present paper is related closely to the dynamics of special relativity, in other words, the particle velocity ( rather than the group velocity) of light ( photons ) propagating through the state of negative temperature may exceed $c$ of the photon in a vacuum; the negative effective rest mass term of light results from the state of negative absolute temperature that is caused by the electric anti-shielding effect ( due to the instantaneous reversals of external electric fields ).

\section{ANTI-SHIELDING EFFECT OF ELECTRIC DIPOLES IN STATE OF NEGATIVE TEMPERATURE}

The potential energy of the electric dipole moment $p_{0}$ in the exterior electric field $E$ is $\varepsilon_{i}=\frac{p_{0} D \cos \theta_{i}}{\epsilon \epsilon_{0}}$, where $\epsilon$ and $\epsilon_{0}$ are respectively the relative permittivity of dielectric and the permittivity of free space; $D$ stands for the electric displacement vector. $\theta_{i}$ is taken to be in the region $0 \leq \theta_{i}<\frac{\pi}{2}$. We regard the continuous angle $\theta$ between dipole moment and exterior field as the discrete $\theta_{i}$ in order to simplify the following calculations. This means the index $i$ ranges from 1 to $\infty$. Let $N_{i}^{+}$and $N_{i}^{-}$respectively denote the occupation numbers of the electric dipoles with the potential energy $\varepsilon_{i}$ and $-\varepsilon_{i}$. Thus the total number $N$ of electric dipoles in the dielectric medium and the total potential energy $U$ of these dipoles in exterior field are given as follows

$$
N=\sum_{i}\left(N_{i}^{+}+N_{i}^{-}\right), \quad U=\sum_{i}\left(N_{i}^{+}-N_{i}^{-}\right) \varepsilon_{i}
$$


$N_{i}^{+}$and $N_{i}^{-}$are solved in what follows. For convenience, we set $N_{i}^{+}=\frac{1}{2}\left(a_{i}+b_{i}\right)$ and $N_{i}^{-}=\frac{1}{2}\left(a_{i}-b_{i}\right)$, and then we have $a_{i}=N_{i}^{+}+N_{i}^{-}, \quad b_{i}=N_{i}^{+}-N_{i}^{-}$and $N=\sum_{i} a_{i}, \quad U=\sum_{i} b_{i} \varepsilon_{i}$. It is apparent that $N$ can be rewritten

$$
N=N \frac{\sum_{i} c_{i}}{\sum_{j} c_{j}}+\sum_{i} f_{i}
$$

with $\sum_{i} f_{i}=0$. Since $N=\sum_{i} a_{i}$, it follows that $a_{i}=N \frac{c_{i}}{\sum_{j} c_{j}}+f_{i}$.

In the similar fashion, $U$ can be rewritten

$$
U=\frac{\sum_{i} d_{i}}{\sum_{j} d_{j}} U+\sum_{i} g_{i} \varepsilon_{i}
$$

with $\sum_{i} g_{i} \varepsilon_{i}=0$. Since $U=\sum_{i} b_{i} \varepsilon_{i}$, it follows that $b_{i} \varepsilon_{i}=\frac{d_{i}}{\sum_{j} d_{j}} U+g_{i} \varepsilon_{i}$ or $b_{i}=\frac{1}{\sum_{j} d_{j}} \frac{d_{i}}{\varepsilon_{i}} U+g_{i}$.

Thus according to the above calculations, the general solution of Eqs. (3.1) is of the form

$$
N_{i}^{+}=\frac{1}{2}\left(\frac{N c_{i}}{\sum_{j} c_{j}}+\frac{1}{\sum_{j} d_{j}} \frac{d_{i}}{\varepsilon_{i}} U+f_{i}+g_{i}\right), \quad N_{i}^{-}=\frac{1}{2}\left(\frac{N c_{i}}{\sum_{j} c_{j}}-\frac{1}{\sum_{j} d_{j}} \frac{d_{i}}{\varepsilon_{i}} U+f_{i}-g_{i}\right) .
$$

The parameters, $c_{i}, d_{i}, f_{i}$ and $g_{i}$, in the general solution (3.4) should be determined by using the physical conditions of the true distribution. It is readily verified that in the two-level system such as spin- $\frac{1}{2}$ model [30,31], the parameters $c_{i}, d_{i}$ and $f_{i}, g_{i}$ in the occupation numbers $N_{i}^{+}$and $N_{i}^{-}$may be $c_{i}=d_{i}=\varepsilon_{i}$ and $f_{i}=g_{i}=0$. Thus the form of $N_{i}^{+}$ and $N_{i}^{-}$is reduced to that of the familiar two-level system. This shows that the above calculations are self-consistent.

According to the Boltzmann's entropy formula ( Boltzmann's relation), one has

$$
S=k_{B} \ln \sum_{i} \frac{N !}{N_{i}^{+} ! N_{i}^{-}} \simeq k_{B}\left[N \ln N-\sum_{i}\left(N_{i}^{+} \ln N_{i}^{+}+N_{i}^{-} \ln N_{i}^{-}\right)\right] .
$$

By using $\frac{\partial}{\partial U}\left(N_{i}^{+} \ln N_{i}^{+}\right)=\frac{1}{2} \sum_{j}^{1} d_{j} \frac{d_{i}}{\varepsilon_{i}}\left(\ln N_{i}^{+}+1\right), \quad \frac{\partial}{\partial U}\left(N_{i}^{-} \ln N_{i}^{-}\right)=-\frac{1}{2} \sum_{j} d_{j} \frac{d_{i}}{\varepsilon_{i}}\left(\ln N_{i}^{-}+1\right)$, one can arrive at

$$
\begin{aligned}
& \frac{1}{T}=\left(\frac{\partial S}{\partial U}\right)_{D}=\frac{k_{B}}{2 \sum_{j} d_{j}} \sum_{i} \frac{d_{i}}{\varepsilon_{i}} \ln \frac{N_{i}^{-}}{N_{i}^{+}} \\
& =\frac{k_{B}}{2 \sum_{j} d_{j}} \sum_{i} \frac{d_{i}}{\varepsilon_{i}} \ln \left\{\frac{\sum_{j}^{N c_{i}}-\frac{1}{\sum_{j} d_{j}} \frac{d_{i}}{\varepsilon_{i}}\left[\sum_{l}\left(N_{l}^{+}-N_{l}^{-}\right) \varepsilon_{l}\right]+f_{i}-g_{i}}{\sum_{j}^{N c_{i}}+\frac{1}{\sum_{j} d_{j}} \frac{d_{i}}{\varepsilon_{i}}\left[\sum_{l}\left(N_{l}^{+}-N_{l}^{-}\right) \varepsilon_{l}\right]+f_{i}+g_{i}}\right\} \text {, }
\end{aligned}
$$

where $D=\epsilon \epsilon_{0} E, \quad \varepsilon_{i}=\frac{1}{\epsilon \epsilon_{0}} p_{0} D \cos \theta_{i}$. Here in our tentative analysis we only consider the isotropic homogeneous medium where the relation $D=\epsilon \epsilon_{0} E$ holds.

In the two-level system, both $c_{i}$ and $d_{i}$ are reduced to $\varepsilon_{i}$. This, therefore, means that in general case, $c_{i}$ and $d_{i}$ may also be the odd functions of $D$ ( or the exterior electric field strength $E$ ). The reason lies in that when the instantaneous inversion of the exterior electric field $E$ takes place, some physical quantities will acquire a minus sign, i. e., $E \rightarrow-E, c_{i} \rightarrow-c_{i}, d_{i} \rightarrow-d_{i}$, but $N_{i}^{+}$and $N_{i}^{-}$are unchanged. From the expressions (3.4), it follows that both $f_{i}+g_{i}$ and $f_{i}-g_{i}$ ( or both $f_{i}$ and $g_{i}$ ) are the even functions with respect to $D$. By using these results, it is shown that the absolute temperature $T$ in the expression (3.6) is the odd function with respect to $D$. This result means that if $E \rightarrow-E$, then $T \rightarrow-T$, namely, the instantaneous inversion of the exterior electric field may cause the state of negative temperature, which is analogous to the case in nuclear spin system e. g., the nuclear spin system of the pure LiF crystal [4-6]. It is therefore apparent that if the swiftly varying exterior electric field inverses all of a sudden and simultaneously the inversion of the dipole moments cannot catch up with the rapid reversal of the exterior electric field, then after it takes the relaxation time $\tau_{1}$ of the dipole-dipole thermal equilibrium depending on the interaction between the dipole moments in the exterior field, the electric-dipole system is therefore getting into the state of negative absolute temperature. If the relaxation time $\tau_{1} \ll \tau_{2}$ with $\tau_{2}$ being the dipole-lattice relaxation time of the thermal equilibrium between dipoles and lattices [4], then this state of negative-temperature dipole system can be readily realized in experiments. Note that this effect via the instant reversals of electric fields can be considered the first anti-shielding effect that acts on the electric dipoles. Its essential feature is characterized by the fact that 
the total potential energy of dipole system in the reversed field is positive ( $U>0$ ). Here one may refer to the case of $U<0$ as the normal shielding effect in electromagnetic media.

It is verified that a second anti-shielding effect due to the state of negative temperature acting upon the electromagnetic wave occurs in the dielectric medium when the light is propagating through it. In general the light field will acquire an effective rest mass term resulting from the shielding effect of electric dipoles. In the case of the instantaneously reversed fields, this mass term in the field equation, however, possesses a minus sign caused by the negative temperature $T$. The equation of the electric field shielded by the electric dipoles may be given $\nabla^{2} \Phi=-\frac{Q}{\epsilon_{0}} \delta n$ with $Q$ being the polarized effective charge in each electric dipole. The right-handed side of this equation arises from the polarization and shielding effect of electric dipoles and $\delta n=n\left[1-\exp \left(\frac{Q \Phi}{k_{B} T}\right)\right]$, where $n=n_{0} \frac{p_{0} E}{k_{B} T}\left[\sinh \left(\frac{p_{0} E}{k_{B} T}\right)\right]^{-1}$ with $n_{0}$ denoting the volume density of the electric dipoles in the dielectric medium. We take the first-order approximation of $\delta n$ and arrive at the result $\delta n \simeq-n \frac{Q \Phi}{k_{B} T}$. Substitution of $\delta n$ into the above electric field equation yields

$$
\nabla^{2} \Phi-\frac{n Q^{2}}{\epsilon_{0} k_{B} T} \Phi=0
$$

It follows that the negative effective rest mass term, $\frac{n Q^{2}}{\epsilon_{0} k_{B} T} \Phi$, in the field equation (3.7) of electric field arises from the anti-shielding effect of electric dipoles due to the state of negative temperature. Further calculation shows that the effective rest mass of light field propagating inside this dielectric may be expressed as

$$
m_{e f f}=\frac{\hbar}{c}\left(\frac{n Q^{2}}{\epsilon_{0} k_{B} T}\right)^{\frac{1}{2}}
$$

Hence we know that if the temperature, $T$, of the dipole system becomes negative, then the effective rest mass ( that accordingly becomes an imaginary number ) of light field may lead to the superluminal light propagation in dielectric media. In the state of low temperature, e. g., $T=1 \mathrm{~K}$, if the instantaneous inversion of the exterior electric field takes place, then the order of magnitude of $\left|m_{e f f}\right|$ may be $10^{-31} \mathrm{~kg}$, where without loss of generality $Q$ and $n$ are taken to be $10^{-20} \mathrm{C}$ and $10^{28} / \mathrm{m}^{3}$, respectively. This means that once the typical light propagation with $\hbar \omega=10^{-19} \mathrm{~J}$ approaches the dielectric medium in the state of negative temperature, the light field obtains a negative rest mass term and therefore will exceed $c$. The similar effect occurs when the supersymmetric partner of the photon, i. e., photinos ( should such exist ) propagates in this anti-shielding dielectric medium and will obtain a negative effective rest mass, which results in the possible negative speed $(\mathbf{v}<0)$ against the propagation direction. It is of interest that the negative speed is faster than the speed exceeding $c$ ( even faster than the positively infinite velocity ), just similar to the fact that the state of negative temperature is hotter than that of positively infinite temperature. In addition, it should be noted that the potential superluminal light propagation in the state of negative temperature ( or the anti-shielding dielectric ) is not at odds with the special relativity nor does it violate the principle of causality, since the above theoretical analysis concerning the superluminal effect is a direct consequence of both the Maxwell's theory and the dynamics of the special relativity. Moreover, this superluminal propagation with particle velocity exceeding $c$ is not strange to us, since there also exists the "tachyon " representation with the four-dimensional momentum squared $p^{\mu} p_{\mu}<0$ in the Poincaré group [7], so that this superluminal motion discussed above is natural from the theoretical point of view. We hold that it deserved the experimental investigation.

How to measure the velocity of this superluminal light propagation in experiment? At low temperature, e. g., $T=1 \mathrm{~K}$, of the dielectric such as the crystals of $\mathrm{HCl}$ and $\mathrm{HF}$, which each dipole respectively possesses $3.62 \times 10^{-30} \mathrm{C} \cdot \mathrm{m}$ and $6.40 \times 10^{-30} \mathrm{C} \cdot \mathrm{m}$ of the dipole moment, in the presence of exterior strong electric field that makes the electric dipoles polarized, the instantaneous reversal of the exterior field may cause the medium into the state of negative temperature. In this situation one may let a light pulse produced at $t=0$ propagate a distance $l_{1}$ and then go through such polarized medium with thickness $l_{2}$. If the light approaches the detector at $t=t_{1}$, then one may take $v=\frac{c l_{2}}{c t_{1}-l_{1}}$ [18], which exceeds $c$. It is worthwhile to point out that in this superluminal phenomenon the group velocity of light field made up of the photons equals the particle velocity of the photons, since the effective rest mass $m_{e f f}=\frac{\hbar}{c}\left(\frac{n Q^{2}}{\epsilon_{0} k_{B} T}\right)^{\frac{1}{2}}$ is independent of the frequency $\omega$ of light field. This, therefore, implies that the group velocity of the light pulse propagating in the anti-shielding dielectric medium may also exceed $c$.

To close this section we briefly consider the anti-shielding effect of thermal electron plasma. According to the Debye's shielding effect [25], the Debye's length is expressed by $\lambda_{D}=\left(\frac{\epsilon_{0} k_{B} T}{N\left(e^{*}\right)^{2}}\right)^{\frac{1}{2}}$ with $N$ being the electron number in per unit volume; hence, the effective rest mass of light field due to this shielding effect is $m_{1}=\frac{\hbar}{c}\left(\frac{N\left(e^{*}\right)^{2}}{\epsilon_{0} k_{B} T}\right)^{\frac{1}{2}}$. If, for example, the plasma is placed in an exterior electric field, here the temperature $T$ in the expression for $m_{1}$ is that 
of the thermal equilibrium caused by potential-potential interaction between electrons themselves ( and ions ) in the electric field, where the particle distribution is in the real space ( associated with the electric potential $\phi(\mathbf{x})$ given by exterior field ), rather than in the velocity space ( associated with the Maxwell's distribution law ). As is presented previously, in cold plasma the light field acquires an effective rest mass whose square is $m_{2}^{2}=\frac{\hbar^{2}}{c^{2}} \frac{N\left(e^{*}\right)^{2}}{\epsilon_{0}} \frac{1}{m c^{2}}$. This result can also apply to the thermal plasma. It thus follows that in thermal electron plasma the total square of rest mass of light field is written in the form

$$
m_{\text {total }}^{2}=m_{1}^{2}+m_{2}^{2}=\frac{\hbar^{2}}{c^{2}} \frac{N\left(e^{*}\right)^{2}}{\epsilon_{0}}\left(\frac{1}{k_{B} T}+\frac{1}{m c^{2}}\right),
$$

where $T$ is the temperature of electrons in potential-potential thermal equilibrium in the real space ( configuration space ). In general this temperature differs from the Maxwell's temperature that depends upon the distribution of particle velocity ( in velocity space). This thermal equilibrium ( Maxwell's velocity distribution ) is often attained by, for instance, the electron-ion collisions where the relaxation time is proportional to $N^{-1}$. This relaxation process to thermal equilibrium can be described by a kinetic equation [32]. Once if the potential-potential relaxation time ( proportional to $N^{-\frac{1}{3}}$ ) is far less than the electron-ion relaxation time, then in the process of the instantaneous reversal of exterior electric field, the potential-potential system is therefore thermally isolated from the Maxwell's velocity distribution system. It follows from (3.9) that if the negative temperature $T$ caused by the instantaneous reversal of exterior field satisfies $0^{-}>T>-\frac{m c^{2}}{k_{B}}$, then the total effective mass squared $m_{\text {total }}^{2}<0$. However, it should be noted that in the case of thermal plasma the negative temperature is not easily achieved, since the potentialpotential system consisting of both quasi-free electrons and ions possesses excessive free energy due to the stored positive potential energy of charged particles in reversed electric field and is therefore not very stable [32]. Only a rather weak perturbation would necessarily destroy this negative-temperature system. For this reason we are therefore not interested in further considering the anti-shielding effect of thermal plasma and the related phenomena.

\section{ANTI-SHIELDING EFFECT AND NEGATIVE TEMPERATURE IN LEFT-HANDED MEDIA}

More recently, a kind of composite media ( the so-called left-handed media ) having a frequency band where the effective permittivity $(\varepsilon)$ and the effective permeability $(\mu)$ are simultaneously negative attracts attentions of many researchers in various fields such as materials science, condensed matter physics, optics and electromagnetism $[3,9,28,33,34]$. Veselago first considered this peculiar medium and showed that it possesses a negative index of refraction [8]. It follows from the Maxwell's equations that in this medium the Poynting vector and wave vector of electromagnetic wave would be antiparallel, i. e., the vector $\mathbf{k}$, the electric field $\mathbf{E}$ and the magnetic field $\mathbf{H}$ form a left-handed system; thus Veselago referred to such materials as "left-handed", and correspondingly, the ordinary medium in which k, $\mathbf{E}$ and $\mathbf{H}$ form a right-handed system may be termed the "right-handed" medium. Other authors call this class of materials "negative-index media (NIM)", "double negative media (DNM) [34]" and Veselago's media. It is readily verified that in such media having both $\varepsilon$ and $\mu$ negative, there exist a number of peculiar electromagnetic properties, for instance, many dramatically different propagation characteristics stem from the sign change of the group velocity, including reversal of both the Doppler shift and Cherenkov radiation, anomalous refraction, modified spontaneous emission rates and even reversals of radiation pressure to radiation tension [9]. In experiments, this artificial negative electric permittivity media may be obtained by using the array of long metallic wires (ALMWs), which simulates the plasma behavior at microwave frequencies, and the artificial negative magnetic permeability media may be built up by using small resonant metallic particles, e. g., the split ring resonators (SRRs), with very high magnetic polarizability [26-29].

In linear media the electric and magnetic polarizations ( per unit volume ) is respectively

$$
\mathbf{P}=(\epsilon-1) \epsilon_{0} \mathbf{E}, \quad \mathbf{M}=(\mu-1) \mathbf{H}
$$

The potential energy density of electric and magnetic dipoles in electromagnetic fields reads

$$
U_{e}=-\mathbf{P} \cdot \mathbf{E}=-(\epsilon-1) \epsilon_{0} \mathbf{E}^{2}, \quad U_{m}=-\mu_{0} \mathbf{M} \cdot \mathbf{H}=-(\mu-1) \mu_{0} \mathbf{H}^{2}
$$

with $\mu_{0}$ being the permeability of free space. It is apparent that in left-handed media where both $\epsilon$ and $\mu$ are negative, the electric and magnetic polarizations, $\mathbf{P}$ and $\mathbf{M}$, are opposite to the electric and magnetic fields, $\mathbf{E}$ and $\mathbf{H}$, thus in general, the potential energy density, both $U_{e}$ and $U_{m}$, are positive, which differs from the cases in the conventional 
media, i. e., the right-handed media. This, therefore, means that left-handed media possess the anti-shielding effect, which results in many anomalous electromagnetic and optical behaviors [9], as is mentioned above.

May the electric and magnetic dipole systems be brought into the state of negative absolute temperature when the electromagnetic wave is propagating in left-handed media? We show that under some restricted conditions, these dipole systems may possess a negative temperature indeed. The detailed analysis is given in what follows:

According to Ramsey's claim [4] that the essential requirement for a thermodynamical system to be capable of a negative temperature is that the elements of the thermodynamical system must be in thermodynamical equilibrium among themselves in order that the system can be described by a temperature at all. For the left-handed media, this may be understood in the sense: the electric and magnetic dipole moments produced by ALMWs and SRRs should be coupled respectively to each other, and this dipole-dipole interactions must be strong and therefore the thermodynamical equilibriums between themselves may be brought about in a short time, which is characterized by the relaxation time $\tau_{1}$. But here both ALMWs and SRRs are macroscopic elements of left-handed medium ( e. g., the length scale of SRRs is $\mathrm{mm}$ ), which implies that there exists a problem as to whether the concept of thermodynamical equilibrium is applicable to these macroscopic elements or not. This problem, however, is not the subject of this section, and it will be published in a separate paper; furthermore, if, for example, we can find a kind of molecular-type SRRs ( i. e., the C- or $\Omega$ - like [35] curved organic molecule that possesses the delocalized $\pi$ bond and can therefore provide a molecular electric current induced by magnetic field of lightwave ) for manufacturing the left-handed media, then the dipole system of this media can be appropriately described by a temperature [4]. So in our tentative consideration in this paper, we can study the anti-shielding effect and negative temperature of left-handed media from the phenomenological point of view, ignoring the detailed information about the elemental atoms which constitute the left-handed media.

In order to generate a state of negative absolute temperature, the following requirement for a thermodynamical system should also be satisfied: the system must be thermally isolated from all other systems, namely, the thermal equilibrium time $\left(\tau_{1}\right)$ among the elements of the negative-temperature system must be short compared to the time during which appreciable energy is lost to or gained from other systems [4]. In left-handed media, the dipole-lattice interaction causes heat to flow from the dipole system ( negative temperature ) to the lattice system ( positive temperature ). If the relaxation time $\left(\tau_{2}\right)$ during which this interaction brings the dipole-lattice system into thermodynamical equilibrium is much greater than $\tau_{1}$, then it may be said that the dipole system is thermally isolated from the lattice system, and the dipole system is therefore capable of a negative temperature. In addition to this condition, there exists another restriction, i. e., the state of negative temperature should be built up within half period of electromagnetic wave propagating inside the left-handed medium, i. e., the relaxation time $\tau_{1}$ should be much less than $\frac{1}{f}$ with $f$ being the frequency of electromagnetic wave, which means that this thermodynamical process caused by dipole-wave interaction can be considered a quasi-stationary process and in consequence the state of negative temperature of dipole system due to dipole-dipole interaction may adiabatically achieve establishment in every instantaneous interval during one period of electromagnetic wave. If this condition is not satisfied, say, the relaxation time $\tau_{1}$ is several times as long as $\frac{1}{f}$, then the negative temperature of dipole systems cannot be achieved since the thermodynamical equilibrium among dipoles induced by the oscillating electromagnetic fields $\mathbf{E}$ and $\mathbf{H}$ may lose its possibility, namely, the concept of thermodynamical equilibrium is not applicable to this non-stationary process; in fact, it should be treated by using non-equilibrium statistical mechanics. In view of above discussions, we conclude that the principal conditions for the electric and magnetic dipole systems of left-handed media possessing a negative temperature may be ascribed to the following two essential requirements

$$
\tau_{1} \ll \tau_{2}, \quad \tau_{1} \ll \frac{1}{f} .
$$

Generally speaking, the first restriction, $\tau_{1} \ll \tau_{2}$, is readily achieved, since in electromagnetic media the dipole-dipole interaction are often much stronger than the dipole-lattice interaction. For example, in some nuclear spin systems such as the pure crystal of $\mathrm{LiF}$ where the negative temperature was first realized in experiments, the relaxation time $\tau_{1}$ of spin-spin process is approximately the period of the Larmor precession of one nucleus in the field of its neighbor and is of the order of $10^{-5}$ second while the relaxation time that depends strongly upon the interaction between the spin system and the crystal lattice is several minutes [4]. In the recent work of designing the structures of more effective left-handed media, use is made of the SRRs of high electromagnetic polarizability [3]. This leads to the very strong dipole-dipole interaction and may achieve the thermodynamical equilibrium of dipole system with shorter relaxation time. If this interaction is rather strong and therefore agrees with the second restriction, $\tau_{1} \ll \frac{1}{f}$ with $f$ being in the region of $\mathrm{GHz}$ [26-28], then the dipole systems of left-handed media may be said to be in the state of negative absolute temperature. 
Since the most conspicuous feature of left-handed media may be the electromagnetic anti-shielding effect, the previous considerations concerning the superluminal light propagation in the instantly varying electric fields based on the viewpoint of effective rest mass can also be applied to the incident electromagnetic wave propagating inside left-handed media. As is stated, in left-handed media many electromagnetic effects such as Doppler shift, Cherenkov radiation and wave refraction are inverted compared with those in ordinary right-handed media. In the similar fashion, it is believed that this superluminal light propagation may also probably occur in left-handed media. Moreover, in some electromagnetic materials, there exists another superluminal pulse propagation that also results from the anti-shielding effect [36,37] ( but rather than from the statistical fluctuations in negative temperature ), since in this media the designed equivalent permittivity and permeability based on the so-called two-time-derivative Lorentz model are simultaneously smaller than the values in free space [36,37]. Ziolkowski has studied the superluminal pulse propagation and consequent superluminal information exchange in this media, and demonstrated that they do not violate the principle of causality [17]. We suggest that all these potential superluminality effects of light propagation in anti-shielding media should be further investigated, particularly in experiments.

\section{CONCLUDING REMARKS}

In electromagnetic systems, the anti-shielding effect is not easily found. The normal shielding effect makes the speed of light wave propagating inside media less than that in a vacuum. In these media the directions of induced electric and magnetic dipoles are parallel ( not antiparallel ) to the electromagnetic fields, and hence the potential energy of these dipoles in the fields are negative as is stated in (4.2). It is for these reasons that in electromagnetic media, systems of negative temperature occur only rarely. In the present paper, for the case of the instantaneous reversal of exterior electric field, we take into consideration the state of negative temperature and the anti-shielding effect of electric dipoles. It follows that if the energy $\varepsilon_{i}$ is changed into $-\varepsilon_{i}$, i. e., once the external field strength changes the sign, after taking the relaxation time the temperature might accordingly become negative (additionally, in the meanwhile the restriction of the dipole-dipole relaxation time being much shorter than the dipole-lattice relaxation time should also be satisfied ). Meanwhile, it follows from the expression for the effective rest mass $m_{e f f}=\frac{\hbar}{c}\left(\frac{n Q^{2}}{\epsilon_{0} k_{B} T}\right)^{\frac{1}{2}}$ that if $T \rightarrow-T$, then $m_{e f f}$ may become imaginary. Hence it is shown from the point of view of effective rest mass that the potentially interesting superluminal light propagation in media may result from this anti-shielding effect of dielectric in the state of negative temperature. According to the conventional viewpoints, the "tachyon " representation in the Poincaré group is often considered the non-physical representation. On the contrary, however, we favor that probably in some certain anti-shielding cases (including the negative-temperature state ), this potential superluminal propagation of light would exist, which deserves the further investigation.

For the case of left-handed media, the anti-shielding effect and the requirements for left-handed media to be capable of negative temperature is analyzed in this paper. In recent investigations regarding the manufacture of left-handed materials, the media capable of negative index of refraction is restricted to the microwave frequency region, since the resonance frequency of SRRs ( and ALMWs ) is low $(\mathrm{GHz})$. If one can find the molecular-type SRRs by certain artificial molecule arrangements, which has the conjugation effect of organic molecules due to the delocalized $\pi$ bond and the consequent high magnetic susceptibility, then the resonance frequency would be readily raised greatly by several orders of magnitude. Based on this, it is of physical significance to consider the interaction of radiation fields with negative-temperature systems in left-handed media. Klimov theoretically studied the processes of spontaneous emission of an atom placed near a body ( sphere ) made of the Left-handed material and placed in the right-handed medium, and showed that these processes drastically differ from those near usual right-handed body [9]. We think if the statistical effect ( including negative temperature ) of left-handed is taken into account, then the results obtained by Klimov should be modified. This modification is under consideration based on the combination of Klimov's formulation and the thermodynamics and statistical mechanics.

Acknowledgements The author thanks Q. Wu, T. Chang and X. M. Wu for their beneficial discussions concerning the superluminal light propagation. I have benefited from useful criticism and advice from C. Alden Mead. My special thanks are due to him also. This project is supported in part by the National Natural Science Foundation of China under the project No. 90101024. 
[1] Camley, R. E. and Mills, D. L., Phys. Rev. B 26, 1280 (1982).

[2] Hartstein, A., Burstein, E., Maradudin, A. A. et al., J. Phys. C 6, 1266 (1973).

[3] Smith, D. R., Padilla, W. J., Vier, D. C. et al., Phys. Rev. Lett. 84, 4184 (2000).

[4] Ramsey, M. F., Phys. Rev. 103, 20 (1956).

[5] Purcell, E. M. and Pound, R. V., Phys. Rev. 81, 279 (1951).

[6] Klein, M. J., Phys. Rev. 104, 589 (1956).

[7] Ramond, P., Field Theory, Massachusetts: Benjamin/Cummings Publishing Company, 15-17 (1981).

[8] Veselago, V. G., Sov. Phys. Usp. 10, 509 (1968).

[9] Klimov, V. V., Opt. Comm. 211, 183 (2002).

[10] Hou, B. Y. and Hou, B. Y., Phys. Ener. Fort. \& Phys. Nucl. (China) 3, 225 (1979).

[11] Shen, J. Q., Zhu, H. Y. and Mao H., J. Phys. Soc. Jpn. 71, 1205 (2002).

[12] Shen, J. Q., Zhu, H. Y. and Shi S. L., Acta Phys. Sin. 51, 536 (2002).

[13] Shen, J. Q., Zhu, H. Y. and Chen P., quant-ph/0205170 < xxx.lanl.gov >.

[14] Akulshin, A. M., Barreiro, S. and Lezama, A., Phys. Rev. Lett. 83, 4277 (1999).

[15] Zibrov, A. S., Lukin, M. D., Hollberg, L. et al., Phys. Rev. Lett. 76, 3935 (1996).

[16] Mitchell, M. W. and Chiao, R. Y., Am. J. Phys. 66, 14 (1998).

[17] Ziolkowski, R. W., Phys. Rev. E 63, 046604 (2001).

[18] Zhou, X. J., Phys. Lett. A 278, 1 (2000).

[19] Nimtz, G., Euro. Phys. J. C 7, 523 (1999).

[20] Nimtz, G. and Heitmann, W., Prog. Quant. Electronics 21, 81 (1997).

[21] Enders, A. and Nimtz, G., J. Phys. I France 2, 1693 (1992).

[22] Heitmann, W. and Nimtz, G., Phys. Lett. A 196, 154 (1994).

[23] Wang, L. J., Kuzmich, A. and Dogariu, A., Nature 406, 277 (2000).

[24] Marangos, J., Nature 406, 243 (2000).

[25] Cairns, R. A., Plasma Physics, Glasgov \& London: Blackie \& Son limited (1985).

[26] Pendry, J. B., Holden, A. J., Stewart, W. J. and Youngs, I., Phys. Rev. Lett. 76, 4773 (1996).

[27] Pendry, J. B., Holden, Robbins, D. J. and Stewart, W. J., J. Phys. Condens. Matter 10, 4785 (1998).

[28] Pendry, J. B., Holden, Robbins, D. J. and Stewart, W. J., IEEE Trans. Microwave Theory Tech. 47, 2075 (1999).

[29] Maslovski, S. I., Tretyakov, S. A. and Belov, P. A., Inc. Microwave Opt. Tech. Lett. 35, 47 (2002).

[30] Datta, N., et al., Phys. Rev. A 40, 526 (1989)

[31] Mizrahi, S. S., Phys. Lett. A 138, 465 (1989).

[32] Alexandrov, Bogdankevich, L. S. and Rukhadze A. A., Priciples of Plasma Electrodynamics, Berlin: Springer-Verlag (1984).

[33] Shelby, R. A., Smith, D. R. and Schultz, S., Science 292, 77 (2001).

[34] Ziolkowski, R. W., Phys. Rev. E 64, 056625 (2001).

[35] Saadoun, M. M. I. and Engheta, N., Microwave Opt. Tech. Lett. 5, 184 (1992).

[36] Ziolkowski, R. W. and Auzanneau, F., J. Appl. Phys. 82, 3192 (1997).

[37] Ziolkowski, R. W. and Auzanneau, F., J. Appl. Phys. 82, 3195 (1997). 